\title{
The Use of a Portable Computer for Real-time Recording of Observations of Grazing Behavior in the Field
}

\author{
MONTAGUE W. DEMMENT AND GREGORY B. GREENWOOD
}

\begin{abstract}
A real-time system for recording grazing behavior in the field is described. A NEC 8201 a portuble computer was programmed to allow the incidence, duration, and sequence of behaviors to be recorded. This system was used in the field to record bites, chews, steps, and swallows of grazing cattle. Data files were analyzed to determine biting, stepping, and swallowing rates as well as bites per feeding station. Durations of all activities can be summarized. This system permits the observer to record accurately feeding behavior and reduce significantly the time and cost of processing data.
\end{abstract}

Ingestive behavior has recently received attention because of a growing appreciation of the complexities of the relationship between the animal and its food supply (Hodgson 1985). Simple measures of the sward or characterizations of forage-on-offer explain only part of the link between plant and animal production. Additional behavioral information is needed to link the animal and its food supply. This approach requires the detailed and accurate recording of behavior.

Observers, limited by recording techniques, frequently tally behaviors over a prescribed period (Hodgson 1982) or record activity by scan samples (Altmann 1974). These data lose the sequence of behavior and suffer from the problems of frequency measures (discussed by Altmann 1974). Furthermore, becausc more than one behavior (e.g., bites or steps) can rarely be recorded simultaneously by one observer, these data cannot be used to examine accurately the relationship between behavioral measures. If observers attempt to record sequential data by hand, they generate long data sheets that require extensive data entry. We report on an ecoding system to record behaviors rapidly from observation in a form that can be directly transferred to a minicomputer.

\section{Methods}

A portable lap-top computer (NEC 8201a), equipped with $40 \mathrm{~K}$ RAM, was affixed to a metal plate. In order to adapt the computer for field use, shoulder straps were attached to the plate to allow typing by a standing observer. Software for data entry was written in $\mathrm{N}_{82}$-BASIC. A cassette tape recorder was used to store files in the field. Data were dumped from the tape recorder through the NEC to our Digital Equipment minicomputer (PDP 11-34) and summarized by programs written specifically to analyze these files.

\section{Data Entry and Analysis}

Data format follows the general form employed by Stephenson and Roberts (1977).A line starter key is typed to capture the time for the onset, termination, or incidence of a behavior. Line starters are a set of keys (any symbol, letter, or number can be chosen in the program) which start a new line. The time is captured and entered automatically at the beginning of that record (Table 1). All keystrokes entered after a line starter and before the next line starter become part of the record of the last line starter. For example, in our data the symbols for a bolus swallowed (during esophageal fistula collections) and steps are line starters (Table 1). Bites are not line starters and for each bite the key is typed once. To calculate the duration of time at a feeding station (Ruyle and Dwyer 1985), one subtracts the time between steps where bites occur. To determine the number of bites per feeding station, bites between sequential steps are counted.

\footnotetext{
Authors are assistant professor and graduate fellow, Department of Agronomy and Range Science, University of California, Davis 95616.

Manuscript accepted 5 January 1987.
}

Table 1. Printout of a typical record of a steer grazing a ryegrass pasture. each time count is .1 sec.

\begin{tabular}{|c|c|c|}
\hline $\begin{array}{l}\text { Time } \\
\text { Count }\end{array}$ & Code & Translation: \\
\hline 0 & ID860516 & DATE: $5-16-86$ \\
\hline 0 & !T14504! & TIME: $14: 50: 41$ \\
\hline 125 & !A22 & animal $\# 22$ \\
\hline 315 & $<$ FFFFFFFFFFF & begin record, bites 13 \\
\hline 584 & /FFFFFF & $\begin{array}{l}\text { step, } 6 \text { bites, new feeding station } \\
\text { (FS) }\end{array}$ \\
\hline 687 & /FFFFF & step 5 bites, new FS \\
\hline 770 & +FFFF & bolus swallowed, 4 , bites \\
\hline 857 & /FFFFFFF & step, 7 bites, new FS \\
\hline 978 & /FFF & step, 3 bites, new FS \\
\hline 1041 & *UP & head up \\
\hline 1058 & ${ }^{*} \mathbf{F}$ & head down, 1 bite \\
\hline 1153 & $+\mathrm{FFF}$ & bolus swallowed, 3 bites \\
\hline 1286 & *UP & head up \\
\hline 1399 & $*$ & head down \\
\hline 1411 & $+\mathbf{F F}$ & bolus swallowed, 2 bites \\
\hline 1424 & /FFFFF & step, 5 bites, new FS \\
\hline 1490 & *UP & head up \\
\hline 1562 & *FFFFF & head down, 5 bites \\
\hline 1618 & $/ \mathrm{FFF}$ & step, 3 bites, new FS \\
\hline 1711 & /FFFFFFFF & \\
\hline 1800 & Cl & comment 1 on voice tape \\
\hline 1867 & $/ \mathbf{F}$ & $\bullet$ \\
\hline 1888 & +FFFFF & $\bullet$ \\
\hline 1971 & /FFFFFFF & $\bullet$ \\
\hline 2134 & + & $\bullet$ \\
\hline 2138 & /FFFFF & $\bullet$ \\
\hline 2262 & UP & $\bullet$ \\
\hline 2479 & $*$ & $\bullet$ \\
\hline 2483 & /FFF & $\bullet$ \\
\hline 2573 & /FFF & $\bullet$ \\
\hline 2722 & $>$ & end data file \\
\hline
\end{tabular}

Some behaviors have a duration too short for analysis of duration such as bites, steps, and chews. These behaviors are referred to as instantaneous and are counted for calculation of their rates. Longer behaviors can have their duration terminated in 2 ways. First, behaviors can begin with a "+" and end with a "-"line starter. Second, some behaviors are always mutually exclusive such as moving and lying. The onset of a movement will always terminate a rest. Programs processing these data can determine the end of an activity based on a matrix of mutually exclusive behaviors.

Some line starters are used to enter data about the context of the file or record. A particular line starter symbol is used to enter the date, subject animal, treatment, and time period. The program automatically captures the time of day from the computer clock at the beginning of the data collection period. Because the computer clock is accurate only to within 1.5 seconds, a timing loop was written in the data entry program to allow accuracy to within.1 sec. While accuracy of the clock time does not represent a major error when the duration of a behavior is long, larger errors are generated when recording common behaviors of short duration. To determine the duration of a behavior, the time count from the line of the 
onset of the behavior is subtracted from the count of the termination line. Other external information can be entered such as pause in data collection, weather information, location of other animals, observer disturbance of the experimental animal, or other comments of interest (Table 1).

While data entry occurs on the computer, we have employed a cassette tape recorder to record a verbal record of the data collection code. This recording provides a backup which, if necessary, can be played and reentered into the computer. This recording is also useful to enter comments, ideas, and observations outside the framework of the code. Indications of particular comments on the cassette tape can be entered into the computer encoding sequence by use of the appropriate line starter (Table 1). The recording also serves as a check of the accuracy of the encoded behavioral sequence. Listening to the tape while reading the computer printout of the file permits errors to be identified and subsequently edited.

Our data recording format is one of a number of possible codes. The format is designed to be flexible and could be used for scan as well as focal samples of behavior. New analysis programs for other types of data would be required, but for most cases these are not complex.

Our analysis program for these files calculates the following: (1) total steps in the file, (2) bites/step, average and variance, (3) chews/step, average and variance, (4) total bites, (5) total chews, (6) frequency distribution of bites/step, (7) frequency distribution of bites/feeding station.

\section{Discussion}

Two problems arose in the use of the NEC computer. Direct sunlight in combination with high ambient temperatures often caused the liquid crystal display to cease functioning. However, while the observer could no longer read on the screen what was typed on the computer, the data entry still occurred. This problem should be of little consequence for the trained observer, who seldom looks at the display while recording data. The second problem is a lack of memory capacity. Although the data collection program produces machine language files, which occupy less memory than text files, memory capacity is limited to approximately 30-45 min data collection. Frequent dumping of files onto a cassette tape is required. This procedure is time consuming and inconvenient if frequent or continuous sampling is the goal. A new disk system, recently available for the NEC, which produces PC compatible files greatly reduces this problem.

The NEC, while having some drawbacks as mentioned above, does have the advantage of being relatively inexpensive when compared to other portable real-time data recorders. The NEC at approximately $\$ 350$ is several thousand dollars cheaper than other systems. These systems include data analysis programs in the field unit. While this may be attractive in some instances, it makes the loss of a unit quite costly. Our development approach has been to try to reduce the cost of the field units as much as possible and put the costly components in the central computer.

\section{Literature Cited}

Altmann, J. 1974. Observational study of behavior: sampling methods. Behavior 48:1-48.

Hodgson, J. 1982. Ingestive behavior. p. 113-138. In: J.D. Leaver (ed.) Herbage intake handbook. British Grassland Soc. Hunley, U.K.

Hodgson, J. 1985. The control of herbage intake in the grazing ruminant. proc. Nutr. Soc. 44:339-346.

Ruyle, G.B. and D.D. Dwyer. 1985. Feeding stations of sheep as an indicator of diminished forage supply. J. Anim. Sci. 61:349-353.

Stephenson, G.B. and T.S. Roberts. 1977. The SSR system 7: a general encoding system with computerized transcription. Beh. Res. Meth. Instr. $9: 434-441$. 